\title{
Convective cells of internal gravity waves in the earth's atmosphere with finite temperature gradient
}

\author{
O. Onishchenko ${ }^{1,2}$, O. Pokhotelov ${ }^{1}$, and V. Fedun ${ }^{3}$ \\ ${ }^{1}$ Institute of Physics of the Earth, 123995, 10 B. Gruzinskaya str., Moscow, Russia \\ ${ }^{2}$ Space Research Institute, 117997, 84/32 Profsoyuznaya str., Moscow, Russia \\ ${ }^{3}$ Dept. of Automatic Control and Systems Engineering, The University of Sheffield, Mappin Street, Sheffield, S1 3JD, UK
}

Correspondence to: O. Onishchenko (onish@ifz.ru)

Received: 1 February 2013 - Revised: 15 February 2013 - Accepted: 22 February 2013 - Published: 7 March 2013

\begin{abstract}
In this paper, we have investigated vortex structures (e.g. convective cells) of internal gravity waves (IGWs) in the earth's atmosphere with a finite vertical temperature gradient. A closed system of nonlinear equations for these waves and the condition for existence of solitary convective cells are obtained. In the atmosphere layers where the temperature decreases with height, the presence of IGW convective cells is shown. The typical parameters of such structures in the earth's atmosphere are discussed.
\end{abstract}

Keywords. Meteorology and atmospheric dynamics (Middle atmosphere dynamics)

\section{Introduction}

The study of the lithosphere and neutral atmosphere/ionosphere coupling is the fundamental problem of geophysics. According to modern concepts the mechanism of lithosphere-ionosphere interaction is closely related to the internal gravity waves (IGWs). In the atmosphere of the earth, the internal gravity waves are generated by various terrestrial processes. For example, thunderstorms, volcanic eruptions, tsunami, typhoons, zonal winds, and earthquakes can be a strong sources of such waves (see e.g. Kazimirovsky et al., 2003; Occhipinti and Lognonné, 2008; Varney et al., 2009; Grimalsky et al., 2010; Rozhnoi et al., 2012, for details). It was shown (see e.g. Hocking, 1996; Fritts and Alexander, 2003; Liu and Gardner, 2004; Lu et al., 2009) that, in an atmosphere in which the density decreases with height, the internal gravity waves propagate to high altitudes with increasing of their amplitude and, therefore, can perturb the ionosphere. Satellite and ground-based electromagnetic sounding of the neutral atmosphere and lower ionosphere has shown a strong connection between terrestrial catastrophes and perturbations of these layers. Therefore, the problem of forecasting and monitoring of terrestrial events by electromagnetic sensing is closely linked with the problem of IGW propagation.

Generation of nonlinear structures may limit the growth of IGW perturbations. The parametric generation of zonal structures by small-scale weakly turbulence of IGW in the atmosphere has been investigated previously by Horton et al. (2008) and Onishchenko and Pokhotelov (2012). Generated zonal wind is a self-regulating system of shear flows, significantly weakening the transport processes in the vertical direction. This mechanism provides an effective channel of energy transfer from the small-scale turbulence region to the region of IGW global convective motions and plays an important role in the regularization of atmospheric turbulence. Such a process is the paradigm of the inverse cascade in turbulent two-dimensional theory of anisotropic turbulence as a result of the formation of a regular large-scale structure from the small-scale chaos.

Nonlinear IGWs can also exist in the form of the vortex structures (see Stenflo, 1987, 1990, 1991; Stenflo and Shukla, 2009, for example). In these works, authors have shown that horizontal velocity of vortices should be of the order or greater than the speed of sound.

The finite vertical gradient of temperature determines dynamics of the vertical transport and convective stability of the atmosphere. The stratified atmosphere is stable, if the square of height-averaged Brunt-Väisälä frequency $\omega_{g}$, i.e.

$$
\omega_{g}^{2}=g\left(\frac{\gamma-1}{\gamma H}+\frac{1}{T} \frac{d T}{d z}\right)
$$




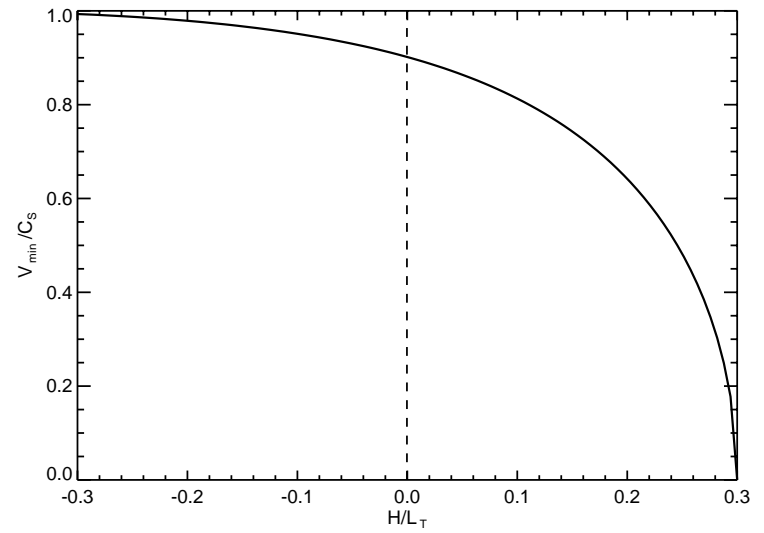

Fig. 1. The normalized minimum speed of vortices $\left(v_{\min } / c_{S}\right)$ as a function of the dimensionless parameter $H / L_{T}$.

is greater than zero. Here, $g$ is the gravitational acceleration, $d / d z$ is the derivative in height, $\gamma$ is the ratio of specific heats, $H$ is the local scale of height of the atmosphere and $T$ is an unperturbed temperature. Usually in the earth's atmosphere $\omega_{g}^{2}$ is positive. But, due to time dependence of temperature gradient it may be negative in some layers. In the regions where the temperature decreases with height and the square of height-averaged Brunt-Väisälä frequency is negative, the powerful vertical convective transport occurs. The condition $\omega_{g}^{2}<0$ corresponds to the absolute instability of IGWs; i.e. frequencies of waves are imaginary.

Nonlinear IGWs in the atmosphere with a finite temperature gradient were investigated previously by Jovanović et al. $(2001,2002)$. Authors have shown that many different types of vortex structures can exist in the atmosphere layers where the condition $\left(d^{2} \rho_{0} / d z^{2}\right)^{1 / 2} \gg d \rho_{0} / d z$ is satisfied. Here, $\rho_{0}$ is the unperturbed density.

In this paper we present a new theoretical study of the nonlinear vortex structures (i.e. convective cells) of IGWs in the earth's atmosphere layers where density and temperature vary in height according to the exponential law. The vertical temperature gradient is taken into account, and the vortex structures have been studied not only in the stably stratified atmosphere, but also in the case of unstable stratified atmosphere. The paper is structured as follows. In Sect. 2 we derive the close system of the nonlinear equations for IGWs. Section 3 is devoted to the stationary vortex solutions and criteria for existence of such structures. Our discussion and conclusions are found in Sect. 4.

\section{The reduced equations for the finite amplitude IGWs}

In the current study we neglect the influence of dissipative processes, e.g. viscosity, thermal conductivity, heat flow from outside, friction, etc. We begin with the hydrodynamic equations in the local Cartesian coordinate system $(x, y, z)$, where the axis $z$ coincides with the vertical. To investigate the non- linear dynamics of low-frequency IGWs, we use conservation equation of momentum

$\rho \frac{d \boldsymbol{u}}{d t}+\nabla p-\rho \boldsymbol{g}=0$

and equation for transport of potential temperature,

$\frac{d \theta}{d t}=0$.

Here $\theta=p^{1 / \gamma} / \rho$ is the potential temperature; $\rho$ and $p$ are the density and pressure, respectively; $d / d t=\partial / \partial t+\boldsymbol{u} \cdot \boldsymbol{\nabla}$ is the Euler (convective) time derivative; $\boldsymbol{u}$ is the velocity of matter; $\boldsymbol{g}=-g \hat{z}$ is the gravity acceleration and $\hat{z}$ is the unit vector of the local Cartesian coordinate system $(x, y, z)$ which is directed along the vertical. To describe the twodimensional motion in the gravitational waves, assume that $\boldsymbol{u}=(u, 0, w)$, where $u=-\partial \psi / \partial z$ and $w=\partial \psi / \partial x$. Here, $\psi(t, x, z)$ is the stream function.

Assuming a weak perturbation, the density $\rho$ and pressure $p$ can be written as follows: $\rho=\rho_{0}(z)+\tilde{\rho}(t, x, z)$, $p=p_{0}(z)+\tilde{p}(t, x, z)$, where $\rho_{0}$ and $p_{0}$ are the unperturbed density and pressure, with $\tilde{\rho}$ and $\tilde{p}$ the corresponding disturbances, $|\tilde{\rho}| \ll \rho_{0}$ and $|\tilde{p}| \ll p_{0}$. As a result, from Eqs. (2) and (3) one obtains a system of two nonlinearly coupled equations:

$$
\begin{array}{r}
\frac{\partial}{\partial t}\left(\nabla^{2} \psi+\frac{d \ln \left(\rho_{0}\right)}{d z} \frac{d \psi}{d z}\right)+J\left(\psi, \nabla^{2} \psi\right) \\
=-\frac{\partial \chi}{\partial x}+\frac{1}{\rho_{0}^{2}} J(\tilde{\rho}, \tilde{p})
\end{array}
$$

and

$$
\frac{\partial \chi}{\partial t}-\omega_{g}^{2} \frac{\partial \psi}{\partial x}+\{\psi, \tilde{\rho}\}=0
$$

Here $J(a, b)=(\partial a / \partial x) \partial b / \partial z-(\partial a / \partial z) \partial b / \partial x$ is the Jacobian, $\nabla^{2}=\partial^{2} / \partial x^{2}+\partial^{2} / \partial z^{2}$, and $\chi=g \tilde{\rho} / \rho_{0}$ is the normalized density perturbation. In an unperturbed atmosphere the pressure decreases exponentially with height; i.e. $p_{0}\left(z-z_{0}\right)=p_{0}\left(z_{0}\right) \exp \left[-\left(z-z_{0}\right) / H\right]$, where $H=$ $c_{S}^{2} / \gamma g$ is the scale height, and $c_{S}=\left(\gamma p_{0} / \rho_{0}\right)^{1 / 2}$ is the speed of sound. Next, let us define the unperturbed temperature as a function of height as $T\left(z-z_{0}\right)=$ $T\left(z_{0}\right) \exp \left[-\left(z-z_{0}\right) / L_{T}\right]$, where $L_{T}$ is the characteristic scale of the vertical temperature gradient. Note that, in the layers of the atmosphere where the temperature decreases with height, $L_{T}>0$; in an inversion layer, $L_{T}<0$. According to the ideal gas law, i.e. $p / \rho T=$ constant, we obtain the expression for the unperturbed density,

$\rho_{0}\left(z-z_{0}\right)=\rho_{0}\left(z_{0}\right) \exp \left[-\left(z-z_{0}\right) / L_{\rho}\right]$,

and the relation between the characteristic scale of the vertical density gradient $\left(L_{\rho}\right)$, the scale height $(H)$ and the characteristic scale of the vertical temperature gradient $\left(L_{T}\right)$ :

$1 / L_{\rho}=1 / H-1 / L_{T}$. 

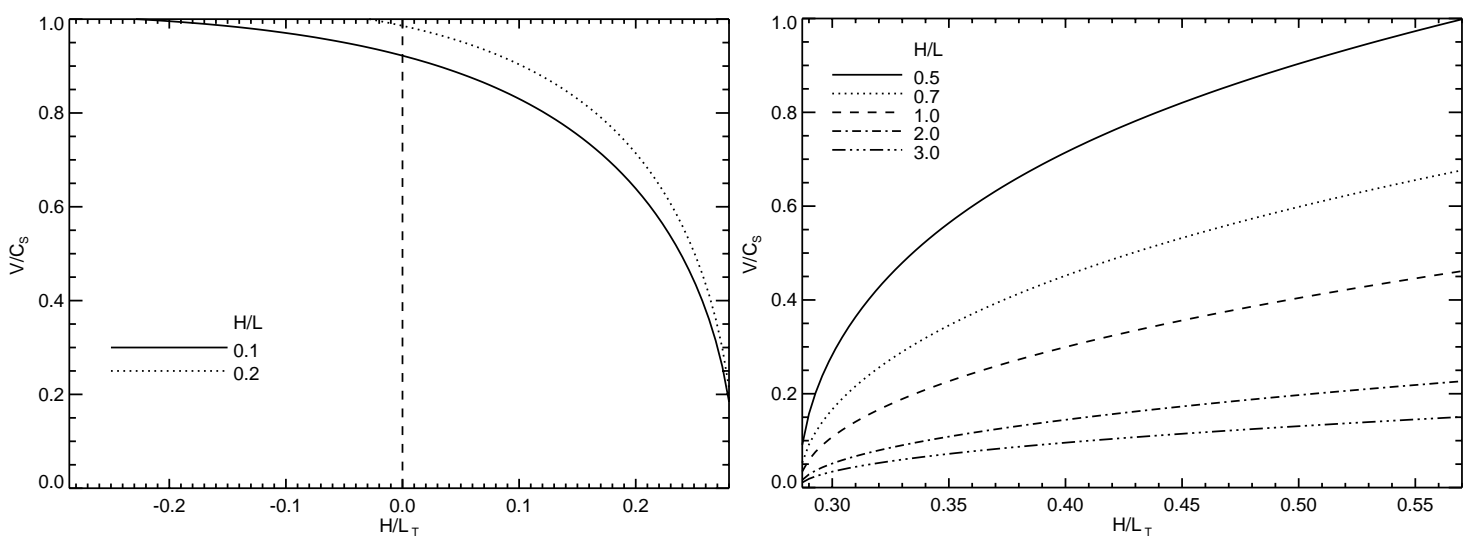

Fig. 2. The normalized horizontal speed of vortex $\left(v / c_{S}\right)$ as a function of the dimensionless parameter $H / L$ and $H / L_{T}$. The vortex speeds have been plotted by assuming the applicable parameters for the stable and unstable stratified atmospheres (see the left and right panels, respectively).

Next, by using the substitution $\psi=\hat{\psi}(t, x, z)$ $\exp \left[\left(z-z_{0}\right) / 2 L_{\rho}\right]$, and $\chi=\hat{\chi}(t, x, z) \exp \left[\left(z-z_{0}\right) / 2 L_{\rho}\right]$, Eqs. (4) and (5) can be represented in the form

$\frac{\partial}{\partial t}\left(\nabla^{2} \hat{\psi}-\frac{\hat{\psi}}{4 L_{\rho}^{2}}\right)+J\left(\hat{\psi}, \nabla^{2} \hat{\psi}\right)=-\frac{\partial \hat{\chi}}{\partial x}$,

and

$\frac{\partial \hat{\chi}}{\partial t}-\omega_{g}^{2} \frac{\partial \hat{\psi}}{\partial x}+J(\hat{\psi}, \hat{\rho})=0$.

This closed system of Eqs. (6) and (7) can be used for numerical simulations of the nonlinear dynamics of IGWs in the earth's atmosphere with a finite vertical temperature gradient. In the limit of an isothermal atmosphere, i.e. if $L_{T} \gg H$, the system of Eqs. (6) and (7) coincides with the system of equations studied previously by Horton et al. (2008); Onishchenko and Pokhotelov (2012); Kaladze and Tsamalashvili (1997). From Eqs. (4) and (5) follows the law of conservation of wave energy $\partial E / \partial t=0$, where

$E=\frac{1}{2} \int \rho_{0}\left[(\nabla \psi)^{2}+\frac{\psi^{2}}{4 L_{\rho}^{2}}+\frac{\chi^{2}}{\omega_{g}^{2}}\right] d x d z$.

Equation (8) shows that, in the case of an unstable stratified atmosphere, the energy can be negative.

\section{Convective cells}

To find a stationary vortex solution in a coordinate system that is moving with the velocity $v$ along the $\mathrm{x}$-axis, let us introduce the new variable $\eta=x-v t$. Under this coordinate transformation the system of the two Eqs. (6) and (7) can be reduced to a single equation:

$J_{1}\left(\nabla^{2} \hat{\psi}-\Lambda \hat{\psi}, \hat{\psi}-v z\right)=0$, where $J_{1}(a, b)=(\partial a / \partial \eta) \partial b / \partial z-(\partial a / \partial z) \partial b / \partial \eta$ and

$\Lambda=\frac{1}{4 L_{\rho}^{2}}-\frac{\omega_{g}^{2}}{v^{2}}$.

A similar equation has been studied analytically and numerically; see e.g. Onishchenko et al. (2008) and references therein. It has been shown that the solution of Eq. (9) can be found in the form of dipole vortices. In the outer region of the vortex condition,

$\nabla^{2} \hat{\psi}-\Lambda \hat{\psi}=0$

is satisfied. Solitary vortices in the atmosphere with a finite vertical temperature gradient exist if $\Lambda>0$. From Eq. (10) one can obtain the relation that connects the characteristic scale of the vortex (convective cell) $L=\Lambda^{-1 / 2}$ with the typical atmospheric parameters scales $L_{T}, H$ and the horizontal velocity of the vortex $v$ :

$\frac{H}{L}=\frac{1}{2}\left[\left(1-\frac{H}{L_{T}}\right)^{2}-\frac{4 c_{S}^{2}}{\gamma v^{2}}\left(\frac{\gamma-1}{\gamma}-\frac{H}{L_{T}}\right)\right]^{1 / 2}$.

The right term of Eq. (12) should be real. Therefore, in the atmosphere layer with decreasing temperature, the vortex velocity should be limited from below. From here we obtain the minimum possible value of the vortex velocity:

$\frac{v_{\min }^{2}}{c_{S}^{2}}=\frac{4}{\gamma}\left(\frac{\gamma-1}{\gamma}-\frac{H}{L_{T}}\right)\left(1-\frac{H}{L_{T}}\right)^{-2}$.

Figure 1 and the left panel of Fig. 2 show dependence of the vortex parameters $v_{\min }$ and $v$ in a stable stratified atmosphere with the effective value $\gamma=1.4$ and $H / L_{T}<0.28$. In unstable stratified atmosphere layers when $H / L_{T}>0.28$, there is no limit on horizontal velocity of vortices and the dependence of normalized vortex velocity on parameters $H / L_{T}$ 
and $H / L$ shown in the right panel of Fig. 2. One can see from these figures that the vortex structures with spatial scales $L$ of the order of $H$ can exist in the atmosphere with $H / L_{T}>0.28$. The right panel of Fig. 2 shows that spatial scale of the vortex structures can be smaller than $H$. With the growth of the vortex scale, the vortex speed is growing.

\section{Conclusions}

In this paper we studied the vortex structure of IGWs in the earth's atmosphere with a finite vertical temperature gradient. The possibility of the vortices' existence in the thermospheric layers, with the vertical temperature gradient of the order of the dry adiabatic gradient, has been shown. The condition for the minimum velocity of vortices in such an atmosphere has been obtained. We have found that the horizontal velocity of the vortices is significantly less than the speed of sound. We have investigated IGW vortex structures in the case of an unstable stratified atmosphere, i.e. for $\omega_{g}^{2}<0$. It is shown that in such atmospheric layers (i.e. in the thermosphere) there is no lower limit on the velocity of the vortices.

Acknowledgements. This work was supported by the Russian Fund for Basic Research (grant 11-05-00920) and Program 8 of the Russian Academy Sciences. Also, authors are grateful for the partial financial support from the International Space Science Institute (Bern, Switzerland) through the grant "Large-scale vortices and zonal winds in planetary atmospheres/ionospheres: Theory vs. observations".

Guest Editor M. Balikhin thanks two anonymous referees for their help in evaluating this paper.

\section{References}

Fritts, D. and Alexander, M.: Gravity wave dynamics and effects in the middle atmosphere, Rev. Geophysics, 41, 1-64, 2003.

Grimalsky, V., Kotsarenko, A., Pulinets, S., Koshevaya, S., and Perez-Enriquez, R.: On the modulation of intensity of Alfvén resonances before earthquakes: Observations and model, J. Atmos. Solar-Terr. Phys., 72, 1-6, 2010.

Hocking, W.: Dynamical coupling processes between the middle atmosphere and lower ionosphere, J. Atmos. Terr. Phys., 58, 735$752,1996$.
Horton, W., Kaladze, T., Van Dam, J., and Garner, T.: Zonal flow generation by internal gravity waves in the atmosphere, J. Geophys. Res., 113, A08312, doi:10.1029/2007JA012952, 2008.

Jovanović, D., Stenflo, L., and Shukla, P. K.: Acoustic gravity tripolar vortices, Phys. Lett. A, 279, 70-74, 2001.

Jovanović, D., Stenflo, L., and Shukla, P. K.: Acoustic-gravity nonlinear structures, Nonlin. Processes Geophys., 9, 333-339, doi:10.5194/npg-9-333-2002, 2002.

Kaladze, T. and Tsamalashvili, L.: Solitary dipole vortices in the Earth's ionosphere, Phys. Lett. A., 232, 269-274, 1997.

Kazimirovsky, E., Herraiz, M., and De La Morena, B. A.: Effects on the Ionosphere Due to Phenomena Occurring Below it, Surv. Geophys., 24, 139-184, 2003.

Liu, A. and Gardner, C.: Vertical dynamical transport of mesospheric constituents by dissipating gravity waves, J. Atmos. Sol. Terr. Phys., 66, 267-275, 2004.

Lu, X. Liu, A. Z., Swenson, G., Li, T., Leblanc, T., and McDermid, I. S.: Gravity wave propagation and dissipation from the stratosphere to the lower thermosphere, J. Geophys. Res., 114, D11101, doi:10.1029/2008JD010112, 2009.

Occhipinti, G., Kherani, E. A. and Lognonné, P.: Geomagnetic dependence of ionospheric disturbances induced by tsunamigenic internal gravity waves, Geophysical Journal International, 173, 753-765, 2008.

Onishchenko, O. and Pokhotelov, O.: Generation of zonal structure by internal gravity waves in the Earths Atmosphere, Doklady Earth Sciences, 445, 845-848, 2012.

Onishchenko, O., Pokhotelov, O., and Astafieva, N.: Generation of large-scale eddies and zonal winds in planetary atmospheres, Physics-Uspekhi, 51, 577-589, 2008.

Rozhnoi, A., Shalimov, S., Solovieva, M., Levin, B., Hayakawa, M., and Walker, S.: Tsunami-induced phase and amplitude perturbations of subionospheric VLF signals, J. Geophys. Res. (Space Physics), 117, A09313, doi:10.1029/2012JA017761, 2012.

Stenflo, L.: Acoustic solitary vortices, Phys. Fluids, 30, 3297-3299, 1987.

Stenflo, L.: Acoustic gravity vortices, Phys. Scripta, 41, 641-642, 1990.

Stenflo, L.: Acoustic gravity vortices, Z. Naturforsch., 46, 560-560, 1991.

Stenflo, L. and Shukla, P.: Nonlinear acoustic-gravity waves, J. Plasma Phys., 75, 841-847, 2009.

Varney, R. H., Kelley, M. C., and Kudeki, E.: Observations of electric fields associated with internal gravity waves, J. Geophys. Res. (Space Physics), 114, 2304, doi:10.1029/2008JA013733, 2009. 